\title{
Aplicación de los clorofluorocarbonos y el hexafluoruro de azufre como trazadores hidrogeológicos en los acuíferos carbonatados del Parque Natural del Barranco del Río Dulce (Guadalajara)
}

\author{
Using chlorofluorocarbons and sulphur hexafluoride as \\ hydrogeological tracers in the limestone aquifers of Parque \\ Natural del Barranco del Río Dulce (Guadalajara)
}

\author{
M. Martín-Loeches Garrido ${ }^{1}$, R. Fisher ${ }^{2}$, S. Díaz Alcaide ${ }^{3}$, P. Martínez Santos ${ }^{3}$ \\ 1 Departamento de Geografía y Geología de la Universidad de Alcalá. Unidad docente de Geología. Edificio de Ciencias del \\ Campus Universitario. Ctra. N-II, km. 33,600. 28871 Alcalá de Henares, Madrid, España. Email: miguel.martin@uah.es \\ 2 NB'l 402, Village Trea, Stung MeanChey, MeanChey, Phnom Penh, Camboya \\ 3 Departamento de Geodinámica, Facultad de Ciencias Geológicas, Universidad Complutense de Madrid 28040 Ciudad \\ Universitaria Madrid, España
}

\begin{abstract}
RESUMEN
A partir de la información procedente de los puntos de agua de los acuíferos asociados a los materiales carbonatados jurásicos y cretácicos próximos al Parque Natural del Barranco del Río Dulce (PNBRD) y de su estructura geológica, se ha establecido un modelo conceptual de flujo subterráneo cuya dinámica fue validada con la interpretación de los contenidos en CFCs y $\mathrm{SF}_{6}$, nunca antes utilizados en España con estos propósitos, de algunas muestras situadas en distintas posiciones. En todos los puntos se reconoce la existencia de una fracción de agua infiltrada antes de 1950 que es mayor en las zonas de recarga de los acuíferos de edad Jurásico donde a través del modelo exponencial se llega a edades aparentes de hasta 100 años. La interpretación de los contenidos en CFCs de todas las muestras a partir del modelo de mezcla binaria, refleja edades superiores a los 17 años para la fracción joven que puede corresponder con aquella que circula por los canales de tamaño intermedio entre la matriz rocosa y los conductos kársticos mayores. Aun no perteneciendo a la misma unidad acuífera, los puntos con mayor proporción de aguas "post-1950" se sitúan en los valles fluviales de los macizos cretácicos. Los resultados son coherentes con sistemas acuíferos de escaso gradiente general, por lo elevado de las edades aparentes obtenidas, la existencia de múltiples entradas de agua y de al menos una doble porosidad, elementos establecidos en el modelo conceptual. En los acuíferos de edad Cretácico existen conductos kársticos que elevan el caudal de los manantiales de forma rápida tras las precipitaciones y cuya influencia no se refleja en las muestras estudiadas. Los CFCs pueden ser útiles para indicar la existencia de fenómenos de contaminación urbana actual. Los valores de edad derivados de las concentraciones de $\mathrm{SF}_{6}$ son inferiores a los deducidos con los CFCs por un probable aporte de este gas del sustrato invalidando su uso como trazador. El futuro del uso de los CFCs se encuentra comprometido por la tendencia decreciente de su concentración en la atmósfera, aunque la comparación del CFC-12 con el $\mathrm{SF}_{6}$, allí donde este gas no sufra incorporaciones de origen geológico, mantendrá su utilidad.
\end{abstract}

Palabras clave: Hidrogeología; clorofluorocarbonos; hexafluoruro de azufre; edad aparente del agua; modelo de flujo; doble porosidad.

Recibido el 28 de mayo de 2014 / Aceptado el 14 de noviembre de 2014 / Publicado online el 17 de marzo de 2015

Citation / Cómo citar este artículo: M. Martín-Loeches Garrido et al. (2015). Aplicación de los clorofluorocarbonos y el hexafluoruro de azufre como trazadores hidrogeológicos en los acuíferos carbonatados del Parque Natural del Barranco del Río Dulce (Guadalajara). Estudios Geológicos 71(1): e025. http://dx.doi.org/10.3989/egeol.41787.332.

Copyright: () 2015 CSIC. This is an open-access article distributed under the terms of the Creative Commons Attribution-Non Commercial (by-nc) Spain 3.0 License. 


\section{ABSTRACT}

Based on information from boreholes and springs of the aquifers associated with the Jurassic and Cretaceous carbonate materials near the Natural Park of the Barranco del Río Dulce (PNBRD) a conceptual groundwater flow model has been established whose dynamics was validated with the interpretation of the contents of CFC and $\mathrm{SF}_{6}$, never before used in Spain for these purposes. At all points there is a fraction of pre-1950 water that is greater in the recharge areas of the Jurassic units, where the interpretation of the data via the exponential model reflects ages up to 100 years. The interpretation of the contents of CFCs from binary mixture model reflects ages greater than 17 years for the young fraction that may correspond with water that flow through the spaces of intermediate size between the rock matrix and karst conduits. Although not belonging to the same aquifer unit, the points with the highest proportion of water "post-1950" are located in the river valleys of the Cretaceous units. The results are consistent with the existence of generally poor hydraulic gradients, because of the high apparent ages obtained, the existence of multiple inlets and at least a double porosity. In the Cretaceous aquifers there are karst conduits that raise the flow of springs quickly after rainfall and whose influence is not reflected in the samples. CFCs can be useful to indicate the existence of phenomena of current urban pollution. Age values derived from $\mathrm{SF}_{6}$ concentrations are lower than those derived from CFCs, probable due to contribution of this gas from the rock invalidating its use as tracer. The future use of CFCs is compromised by the decreasing tendency of its concentration in the atmosphere, although the comparison of CFC-113 with SF 6 of no geological origin, will maintain its utility.

Key words: Hydrogeology; chlorofluorocarbons; sulfur hexafloride; apparent age; flow model; double porosity.

\section{Introducción}

Los clorofluorocarbonos (CFCs) son compuestos sintéticos de cloro, carbono y flúor que fueron producidos comercialmente desde la década de los años 30 del pasado siglo para refrigeración y otras aplicaciones industriales. El primero en liberarse a la atmósfera fue el CFC-12, en 1930; desde 1936 se produjo el CFC-11, y finalmente, desde 1944, el CFC-113 (IAEA, 2006). La concentración de CFCs en la atmósfera se incrementó de manera muy notable desde los años 50 hasta el comienzo de los años 90, cuando dejaron de emitirse tras los acuerdos derivados del Protocolo de Montreal. Actualmente, las curvas de concentración de estos gases en la atmósfera se encuentran decreciendo.

Los CFCs se han utilizado con éxito en numerosos estudios como trazadores y para datar aguas subterráneas infiltradas después de 1950 (Darling et al., 2012; Cook \& Solomon, 1995 y 1997; Plummer \& Busemberg, 1999; Busemberg \& Plummer, 1992 y 2000; IAEA, 2006); también para estimar las proporciones de mezcla de agua de diferentes edades en las muestras (Plummer et al., 2003; IAEA, 2006). Indirectamente, estos compuestos se pueden usar para calibrar modelos de flujo y para evaluar la susceptibilidad de una fuente de agua potable a contaminarse (Busenberg \& Plummer, 1992).

El hexafluoruro de azufre $\left(\mathrm{SF}_{6}\right)$ comenzó a emitirse a la atmósfera a partir de 1953 y es un gas con un claro potencial para los mismos propósitos (Busenberg \& Plummer, 2000, 2006 y 2008).
A diferencia de lo que ocurre con los CFCs, la concentración en la atmósfera del $\mathrm{SF}_{6}$ sigue incrementándose, lo que supone un factor importante para el futuro de su uso como trazador.

Ninguno de estos compuestos ha sido utilizado antes en España en el ámbito hidrogeológico.

La datación con CFCs y $\mathrm{SF}_{6}$ se basa en la comparación de las concentraciones de estos elementos en equilibrio en las muestras de agua subterránea, con el contenido histórico de sus concentraciones en la atmósfera. La Fig. 1 muestra los valores de CFC-11,

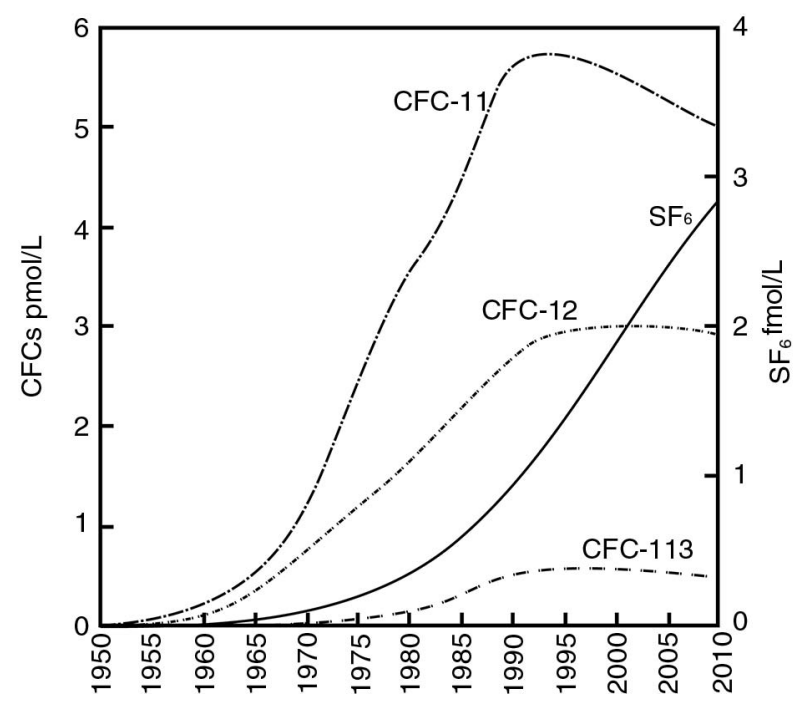

Fig. 1.- Variación de la concentración de CFCs y $\mathrm{SF}_{6}$ en las aguas subterráneas del hemisferio norte para una temperatura de recarga de $10^{\circ} \mathrm{C}$. Datos tomados de la página web del Servicio Geológico de los Estados Unidos. 
CFC-12, CFC-113 y SF 6 que se esperarían en las aguas subterráneas del hemisferio norte recargadas entre 1950 y 2010 a $10{ }^{\circ} \mathrm{C}$ de temperatura y a nivel del mar. Según los datos de esta figura, en condiciones ideales, el agua recargada en 1985 y correspondiente a un modelo de flujo pistón (PFM) (muestra de agua sin mezcla, recogida de un sondeo equipado con una rejilla estrecha en un acuífero detrítico homogéneo e isótropo, o de un manantial somero), debería contener, $4.5 \mathrm{pmol} / 1$ de CFC-11, $2 \mathrm{pmol} / 1 \mathrm{de}$ CFC-12 y 0.3 pmol/1 de CFC-113 y $0.65 \mathrm{fmol} / \mathrm{L}$ de $\mathrm{SF}_{6}$ (ver Fig. 1).

El modelo de flujo pistón (PFM) es aquel en el que no existe mezcla de aguas de diferente procedencia o edad; se suele describir como la situación ideal a la que se llegaría si el agua fluyera a través de un tubo de flujo desde la recarga hasta la descarga sin sufrir ninguna interacción con tubos de flujo contiguos. En estos casos, efectivamente, el agua presentaría una edad uniforme.

Es difícil sin embargo recoger muestras que se ajusten a esas condiciones. El agua que se bombea de un pozo o que emerge en un manantial es normalmente una mezcla de aguas procedentes de diferentes líneas de flujo. Para la interpretación de los contenidos en CFCs y $\mathrm{SF}_{6}$ en estos casos, debemos recurrir a otros modelos; los más utilizados son el modelo de mezcla exponencial (EXM) y el modelo de mezcla binaria (MB) (IAEA, 2006); existen además modelos intermedios, como el flujo pistón exponencial o el de mezcla exponencial (Darling et al., 2012).

En el modelo exponencial, la muestra se interpreta como un número infinito de sub-muestras que presentan una distribución exponencial de edad y se aporta una edad media general. Esta situación puede corresponder con la que sufrirían muestras tomadas de pozos abiertos en una longitud amplia de su tubería en acuíferos detríticos, o en manantiales de descarga regional.

En el modelo de mezcla binaria se considera al agua de la muestra como el resultado de la mezcla entre "agua vieja", o libre de CFCs (recargada anteriormente a 1950) y "agua joven" o con contenido en CFCs (post 1950). Este modelo es uno de los más simples y quizá el más importante en acuíferos fracturados (Gooddy et al., 2006). Las muestras tomadas en acuíferos carbonatados en los que existe una red de canales de flujo preferente junto con una matriz rocosa saturada donde se produce un flujo mucho más lento, podría ajustarse a este modelo.

Existen además algunos factores que deben tenerse en cuenta ya que pueden distorsionar los valores reales de edad. Entre ellos destacan la temperatura y la altitud en la recarga, el espesor de la zona no saturada, la elevada concentración de estos compuestos en el aire urbano, la degradación microbiana - que afecta de manera diferente a cada compuesto-, la absorción de estos compuestos a la fase sólida del acuífero, si este tiene alto contenido en carbono orgánico, y las mezclas de aguas (Darling et al., 2012).

En el presente trabajo se han analizado los contenidos de CFCs y $\mathrm{SF}_{6}$ de aguas subterráneas procedentes de los acuíferos carbonatados de edad Jurásico y Cretácico que se encuentran en el entorno del Parque Natural del Barranco del Río Dulce (PNBRD) en la provincia de Guadalajara (Fig. 2) y que pertenecen a la masa de agua subterránea "030.003 TajuñaMontes Universales" (CHT, 2013) o la Unidad Hidrogeológica homónima 03.02 de IGME (2013), para caracterizar su edad (tiempo transcurrido desde su infiltración hasta su muestreo) como primer objetivo a partir de los diferentes modelos mencionados. Siendo la primera vez que se utilizan en España estos compuestos en el campo de la hidrogeología, otro objetivo del trabajo es valorar su utilidad en acuíferos del centro peninsular.

\section{Material y métodos}

Las muestras fueron recogidas siguiendo a Busenberg et al. (2006). Se tomaron dos muestras por cada punto para confirmar los resultados. Se utilizaron botellas de vidrio de $500 \mathrm{ml}$ (suficiente para realizar todas las determinaciones) que fueron llenadas con el agua problema bombeada a través de un tubo de nylon de $10 \mathrm{~mm}$ de diámetro. Dicha botella debe transportarse al laboratorio en el interior de una cápsula metálica llena también con el agua muestreada.

Durante el muestreo, tanto la botella como la cápsula deben situarse en el fondo de un cubo; se bombea el agua al interior de la botella a través de la manguera de nylon y se deja que fluya hasta que rebose por encima del cubo. Después, en condiciones sumergidas, se cierra la botella y se introduce 


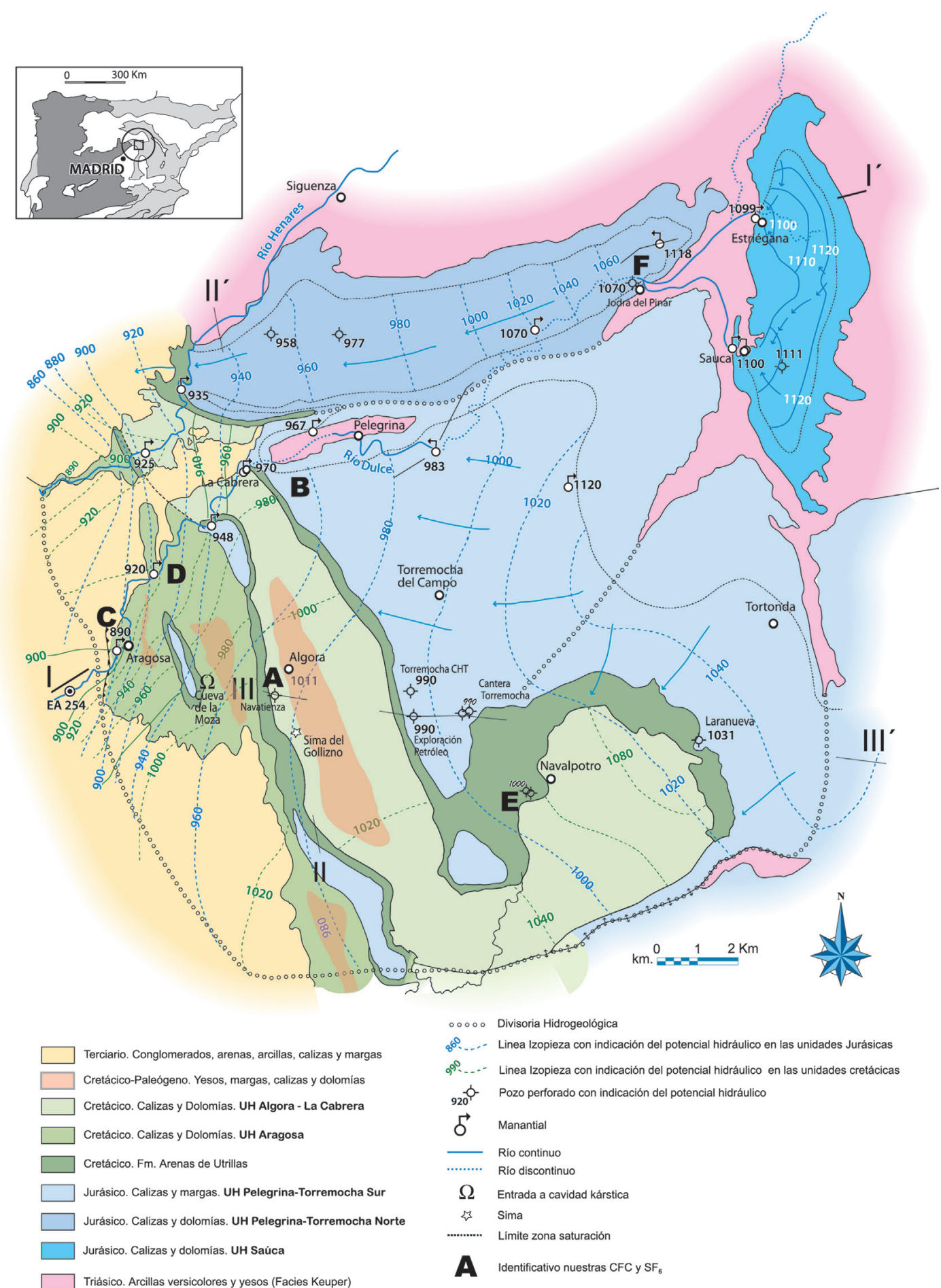

Fig. 2.-Esquema hidrogeológico de la región estudiada. Cartografía geológica basada en Adell et al (1981 a y b), Bascones y Martínez, (1981) y Ríos (2002).

ésta en la cápsula metálica que también debe sellarse a través de una cinta de goma que encaja a presión. Esta técnica de muestreo crea una barrera contra la contaminación del agua de la botella interior. Antes de proceder a tomar una muestra, el equipo era lavado con el agua a muestrear. 
Se debe evitar el contacto del agua con el aire de la atmósfera, ya que de producirse, la muestra se contaminaría. Esto supuso un factor limitante a la hora de seleccionar los puntos de agua ya que muchos no presentaban las condiciones mínimas que garantizasen un completo aislamiento del agua durante el muestreo. Para evitar esta contaminación, dependiendo del tipo de salida del agua, se introducía un extremo del tubo de nylon hasta asegurarse de que no existiera contacto del agua con el aire atmosférico. El otro extremo terminaba en el fondo de la botella de muestras.

Las muestras fueron tomadas en el mes de octubre, tras el periodo de estiaje, para minimizar la influencia de aguas superficiales.

Se tomaron muestras de 6 puntos de agua; su posición se indica en el mapa de la Fig. 2, y en los cortes de la Fig. 3 con las letras identificativas $\mathrm{A}, \mathrm{B}, \mathrm{C}, \mathrm{D}, \mathrm{E}$ y $\mathrm{F}$. Los resultados se presentan en la Tabla 1. Corresponden a tres muestras en el Jurásico carbonatado y tres en el Cretácico, también carbonatado.

Las determinaciones analíticas de CFCs y de $\mathrm{SF}_{6}$ se realizaron por cromatografía de gases obtenidos mediante purga y atrapamiento en el laboratorio del Dr. Oster en Wachenheim, Alemania. Las concentraciones de los gases fueron medidas con un detector de captura de electrones (ECD, en sus siglas en inglés). Una exhaustiva explicación de los métodos de análisis utilizados puede obtenerse en Bullister \& Weiss (1988) y en Busenberg et al. (2006).

Los datos fueron interpretados con la ayuda de la hoja de cálculo que para tal propósito ofrece el Servicio Geológico de los Estados Unidos (USGS, en sus siglas en inglés) en su página web (USGS, 2013). Esta herramienta calcula las edades aparentes del agua a partir de los registros históricos de concentración en la atmósfera en $\mathrm{CFCs}_{\text {y }} \mathrm{SF}_{6}$ según el modelo pistón, el modelo de mezcla binaria y el modelo exponencial. El programa usa los datos de la estación de Niwot Ridge en Estados Unidos que son válidos para todo el hemisferio Norte a excepción de las áreas industriales y urbanas donde existe una mayor concentración de estos compuestos. Los resultados de estas interpretaciones se muestran en la Tabla 2 y la Fig. 5 .

\section{Marco geológico e hidrogeológico y modelo conceptual de flujo de los acuíferos estudiados}

El PNBRD se encuentra en el borde centrooriental de la Cordillera Ibérica, próximo a la Cuenca terciaria del Tajo. La sucesión estratigráfica en esta región está compuesta por materiales triásicos, jurásicos y cretácicos suavemente plegados en la Orogenia Alpina. La Fig. 2 muestra un esquema de localización y un esquema hidrogeológico de la región en el que se señalan las unidades acuíferas que diferencia Matín-Loeches, (2008a) en base a la estructura tectónica, la presencia de la Formación Arenas de Utrillas, del Cretácico, y de las arcillas, limos y yesos en facies Keuper del Triásico, que son materiales de baja permeabilidad.

La erosión ha aislado algunos macizos calcáreos de edad Jurásico, que constituyen unidades hidrogeológicas independientes como la Unidad "Saúca" (Fig. 2). Las unidades Pelegrina-Torremocha ( $\mathrm{N}$ y S) (Jurásico), Algora-La Cabrera (Cretácico) y Aragosa (Cretácico) (Fig. 2), forman el sistema hidrogeológico Pelegrina-La Cabrera-Aragosa de Martín-Loeches (2008 a).

El sustrato Jurásico puede alcanzar hasta 375 metros de potencia de materiales margosos intercalados con calcáreos (Goy et al., 1976). Las margas son predominantes en los 100 metros superiores, aunque culminan la serie jurásica 10 metros de calizas micríticas (Ríos et al., 2002). Es frecuente que las calizas y dolomías que dominan en las unidades inferiores se presenten en estratos finos y medios o en tramos brechoides, oquerosos (carniolas) y con un elevado grado de fracturación y diaclasado. No existen grandes bancos de calizas o dolomías continuas de edad Jurásico a través de los cuales se desarrolle un sistema kárstico; la única forma kárstica reconocible en superficie, salvo pequeños torcales, es el valle ruiniforme que se localiza en el cañón del río Dulce entre Pelegrina y Jodra del Pinar sobre la formación Calizas y Dolomías Tableadas de Adell et al. (1981a) (García Hidalgo et al, 2008) cerca del nivel de base actual representado por el cauce del río Dulce.

Se han inventariado 11 pozos perforados en los que se registra una oscilación piezométrica anual cercana a los 20 metros entre 2008 y 2013. Solo en 5 de ellos existe acceso y posibilidad de realizar 

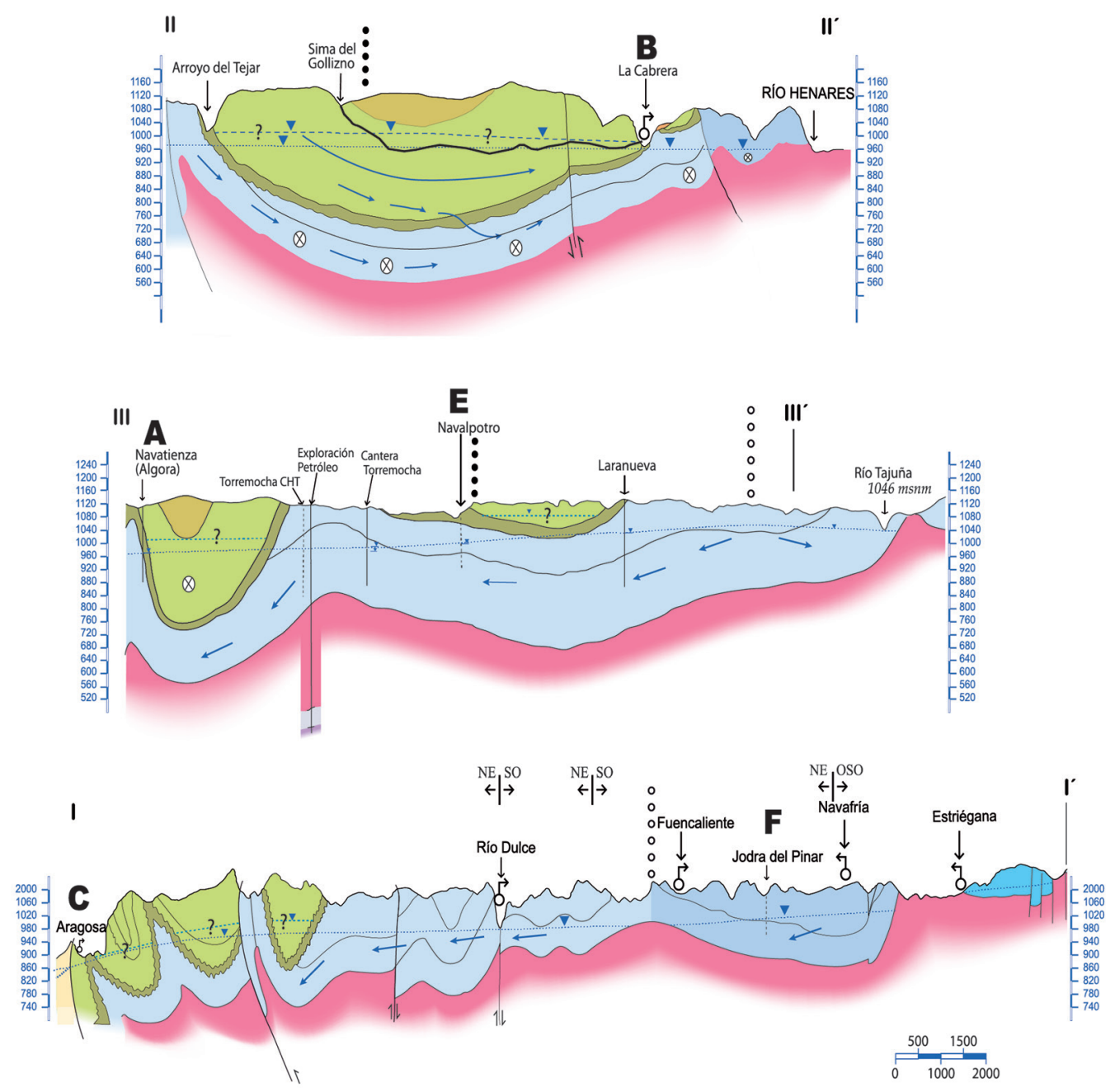

\section{LEYENDA}

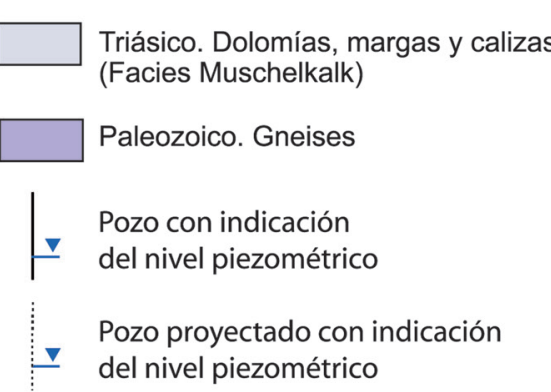

$\bullet \bullet \bullet$ Divisoria Hidrológica

$\lessdot \quad$ Manantial

- $\boldsymbol{\nabla}_{\text {- }}$. Superficie piezométrica en unidades cretácicas

........ Superficie piezométrica en unidades jurásicas

$\longrightarrow$ Probable dirección de flujo subterráneo

Q Flujo transversal a la dirección del perfil

Fig. 3.-Cortes hidrogeológicos indicados en el mapa de la Fig. 2. La leyenda es complementaria a la que acompaña a la Fig. 2. La información de las columnas litológicas procede de empresas privadas y del Archivo Técnico de Hidrocarburos (Ministerio de Industria, Turismo y Comercio de España, 2014).

medidas periódicas y de estos, dos son piezómetros de control de la Confederación Hidrográfica del Tajo (CHT, 2013). Los niveles restantes corresponden a los tomados durante la ejecución de las perforaciones. Los pozos más profundos perforados en estas calizas (entre 200 y 256 metros), raramente resultan negativos 
Tabla 1.-Resultados obtenidos del contenido en $\mathrm{CFCs}_{\mathbf{y}} \mathbf{S F}_{6}$ de las muestras analizadas

\begin{tabular}{|c|c|c|c|c|c|c|c|c|c|c|}
\hline ID & Unidad Hidrogeológica (Edad) & $\begin{array}{l}\text { Localización/ } \\
\text { Tipo de punto }\end{array}$ & Fecha & $\begin{array}{c}\text { Altura de la } \\
\text { recarga }(\mathrm{msnm})\end{array}$ & $\begin{array}{l}\mathrm{T} \text {. en la } \\
\text { recarga }{ }^{\circ} \mathrm{C}\end{array}$ & $\begin{array}{c}\text { Profundidad } \\
\text { ZNS (m) }\end{array}$ & $\begin{array}{l}\text { CFC-11 } \\
(\mathrm{pmol} / \mathrm{l}) \\
\end{array}$ & $\begin{array}{l}\text { CFC-12 } \\
(\mathrm{pmol} / \mathrm{l})\end{array}$ & $\begin{array}{l}\text { CFC-113 } \\
(\mathrm{pmol} / \mathrm{l})\end{array}$ & $\begin{array}{c}\text { SF6 } \\
(\mathrm{fmol} / \mathrm{l})\end{array}$ \\
\hline$A$ & Pelegrina-Torremocha Sur (Ju) & Navatienza/PP & 17.10 .2008 & 1140 & 12 & 118 & $2.0 \pm 0.2$ & $1.0 \pm 0.1$ & $0.2 \pm 0.05$ & $0.7 \pm 0.1$ \\
\hline B & Algora-La Cabrera (Cre.) & La Cabrera/M & 17.10 .2008 & 1140 & 12 & 118 & $5.9 \pm 0.6$ & $1.9 \pm 0.1$ & $0.32 \pm 0.05$ & $1.9 \pm 0.2$ \\
\hline C & Aragosa (Cre.) & Aragosa/M & 17.10 .2008 & 1020 & 14 & 40 & $2.3 \pm 0.3$ & $1.4 \pm 0.1$ & $0.2 \pm 0.05$ & $1.0 \pm 0.1$ \\
\hline D & Aragosa (Cre.) & El Portacho/M & 21.10 .2008 & 1040 & 14 & 40 & $2.4 \pm 0.3$ & $1.4 \pm 0.1$ & $0.21 \pm 0.05$ & $1.1 \pm 0.2$ \\
\hline$E$ & Pelegrina-Torremocha Sur (Ju) & Navalpotro/PP & 17.10 .2008 & 1120 & 12 & 80 & $1.1 \pm 0.2$ & $0.61 \pm 0.05$ & $0.09 \pm 0.05$ & $1.1 \pm 0.2$ \\
\hline $\mathrm{F}$ & Pelegrina-Torremocha Norte (Ju.) & Jodra del Pinar/PP & 21.10 .2008 & 1130 & 13 & 12 & $2.6 \pm 0.3$ & $1.7 \pm 0.1$ & $0.28 \pm 0.05$ & $2.4 \pm 0.3$ \\
\hline
\end{tabular}

ID = Identificativo.

Tabla 2.-Interpretación de los resultados según diferentes modelos

\begin{tabular}{|c|c|c|c|c|c|c|c|c|c|c|c|c|}
\hline \multirow[b]{3}{*}{ ID } & \multicolumn{7}{|c|}{ Flujo pistón } & \multirow{2}{*}{\multicolumn{3}{|c|}{$\begin{array}{c}\text { Modelo exponencial } \\
\text { Edades CFC }\end{array}$}} & \multirow{2}{*}{\multicolumn{2}{|c|}{$\begin{array}{c}\text { Mezcla binaria } \\
\text { CFC113/CFC12 }\end{array}$}} \\
\hline & \multicolumn{5}{|c|}{ Edades CFC } & \multicolumn{2}{|c|}{ Edades SF6 } & & & & & \\
\hline & CFC-11 & CFC-12 & CFC-113 & $\begin{array}{l}\text { Año medio } \\
\text { de la } \\
\text { recarga }\end{array}$ & $\begin{array}{l}\text { Tiempo de } \\
\text { residencia } \\
\text { (años) }\end{array}$ & $\begin{array}{l}\text { Año medio } \\
\text { de la } \\
\text { recarga }\end{array}$ & $\begin{array}{l}\text { Tiempo de } \\
\text { residencia } \\
\text { (años) }\end{array}$ & Trazadores usados & $\begin{array}{l}\text { Tiempo de } \\
\text { residencia } \\
\text { (años) }\end{array}$ & $\begin{array}{l}\text { Desviación } \\
\text { estandard } \\
\text { (años) }\end{array}$ & $\begin{array}{c}\text { Edad } \\
\text { (años) }\end{array}$ & $\begin{array}{c}\text { \% agua "joven" } \\
\text { en mezcla con } \\
\text { CFC } 113\end{array}$ \\
\hline$A$ & 1975 & 1974 & 1983.5 & 1978 & 32 & 1985 & 24 & CFC-12 CFC-113 CFC-11 & 46 & \pm 9 & 17.3 & 46.0 \\
\hline B & Con. & 1985.5 & 1987.5 & 1987 & 23 & 1997 & 12 & CFC-12 CFC-113 & 16 & \pm 1 & 19.8 & 86.2 \\
\hline C & 1977 & 1981 & 1984.5 & 1980 & 29 & 1989 & 20 & CFC-12 CFC-113 CFC-11 & 36 & \pm 6 & 21.8 & 75.1 \\
\hline D & 1978 & 1981 & 1985 & 1981 & 28 & 1990 & 19 & CFC- 12 CFC-113 CFC-11 & 34 & \pm 5 & 21.3 & 74.3 \\
\hline$E$ & 1970.5 & 1970 & 1977.5 & 1973 & 37 & 1989 & 19 & CFC-12 CFC-113 CFC-11 & 100 & \pm 9 & 21.3 & 30.6 \\
\hline $\mathrm{F}$ & 1978.5 & 1984 & 1987 & 1983 & 26 & 2001 & 7 & CFC-12 CFC-113 CFC-11 & 25 & \pm 8 & 19.8 & 79.8 \\
\hline
\end{tabular}

$\mathrm{ID}=$ Identificativo; Con. = Contaminado (valor superior al máximo en la atmósfera). 
aunque aportan caudales siempre inferiores a los $5 \mathrm{l} / \mathrm{s}$. Son frecuentes los manantiales estacionales derivados de la alternancia de calizas y margas situación que define en algunas zonas un nivel piezométrico colgado con interés local (Torremocha del Campo).

No hay evidencias de importantes conductos en las calizas Jurásicas a través de los cuales se defina un flujo veloz del agua subterránea, y divida al conjunto rocoso en grandes bloques. Al menos no se han podido localizar puntos importantes de descarga donde estudiar las variaciones de caudal tras las precipitaciones (Pulido, 2001). Las margas en las unidades geológicas superiores pueden jugar un cierto papel en el almacenamiento del agua que es transferida al medio fisurado inferior donde la transmisión es mayor; en la zona saturada las margas deben retardar la velocidad del flujo. Entre los rangos propuestos para modelos de flujo conceptuales en acuíferos carbonatados por diferentes autores (White, 1969, Atkinson \& Samart, 1981; Smart \& Hobbs, 1986; Quinlan et al., 1992), deducimos que el material de edad Jurásico se situaría entre aquellos en los que domina el flujo difuso con movimiento lento a través del sustrato, comparable al medio de porosidad intergranular con elevada anisotropía y heterogeneidad, que describe para otros macizos carbonatados españoles Pulido, (1998).

La recarga en estos materiales jurásicos es predominantemente difusa, aunque no podemos descartar que una parte se produzca de forma puntual. También pasa agua de las unidades cretácicas a través de las arenas de Utrillas en una cantidad no cuantificada (Martín-Loeches et al., 2011). Su descarga se debe producir en el paraje de los Heros, a $948 \mathrm{msnm}$, y al pie del Mirador de Pelegrina a 983 msnm (ver Fig. 2) con unos $2 \mathrm{hm}^{3}$ anuales en años de precipitación media (Martín-Loeches et al., 2011).

Las líneas isopiezas marcadas en las unidades jurásicas en la Fig. 2 constituyen aproximaciones de la posición del nivel de saturación regional, y son válidas para mostrar la dirección preferente del flujo. $\mathrm{Su}$ trazado marca un gradiente hidráulico próximo a 0.01 en las zonas más alejadas de los ríos Dulce y Henares, que provocaría un flujo en la matriz del sustrato muy lento.

Los materiales carbonatados del Cretácico lo forman hasta 400 metros de calizas y dolomías en bancos de espesor medio bien definidos, a veces masivos. Culminan en algunas zonas del área de estudio con unos 60-100 metros de yesos, margas, calizas y dolomías en tránsito hacia el Paleógeno (Ríos et al., 2002). La proporción de materiales margosos es mucho menor que en los materiales jurásicos; también se aprecia en las calizas del cretácico una menor presencia de fracturación. Existen formas exokársticas en superficie como torcales y simas y se desarrollan cavidades visitables (cueva de la Moza o de La Oliva, ver Fig. 2).

En las unidades cretácicas al sur del cauce del río Henares no existen pozos. Nos consta que algunos intentos de perforación en el Cretácico fueron infructuosos, circunstancia frecuente en macizos calcáreos típicamente kársticos (Pulido, 1998). La mayoría de los puntos del inventario corresponde a manantiales de descarga regional de entre los que destaca el manantial de La Cabrera porque registra un caudal mínimo de $30 \mathrm{~L} / \mathrm{s}$.

La respuesta de los manantiales tras las lluvias es muy rápida y se superpone durante un breve lapso de tiempo a un flujo de base muy continuo y a otro que refleja las lluvias de primavera y otoño. Las estimaciones realizadas en el manantial de La Cabrera (García-Hidalgo et al, 2008) señalan que el flujo de base puede constituir hasta el $50 \%$ de la aportación total $\left(0.85 \mathrm{hm}^{3}\right.$ de $1.4 \mathrm{hm}^{3}$ totales en años de precipitación media). Ensayos con trazadores realizados en las simas cercanas a Algora muestran en el Cretácico velocidades máximas próximas a los $4000 \mathrm{~m} /$ día.

Estos datos señalan que en las unidades cretácicas debe existir un nivel de saturación general, y que coexisten conductos de alta permeabilidad a través de los cuales el agua circula con rapidez y bajo un régimen turbulento, con una red de discontinuidades menores, fisuras, planos de estratificación e incluso poros en la matriz rocosa en la que se produce flujo lento (Worthington, 2007; Ford \& Williams, 2007; Goldscheider \& Drew, 2007; Pulido, 2001). Los bloques entre conductos constituyen el elemento capacitivo mientras que los canales serían el elemento transmisivo.

La recarga de las unidades cretácicas es en gran medida puntual, a través de las simas; en los núcleos sinclinales aflora la unidad superior impermeable (Fig. 2) que forma áreas de recarga alogénicas (Goldscheider \& Drew, 2007). La descarga se produce al rio Dulce en los manantiales próximos a su cauce con $3.15 \mathrm{hm}^{3}$ en años de precipitación media y en parte al río Henares. 
Hemos marcado en la Fig. 2 líneas isopiezas supuestas en las unidades cretácicas que tratan de indicar de manera aproximada la dirección general del flujo subterráneo y su relación con los ríos; no incluye la red de conductos kársticos de mayor velocidad, ya que no se ha podido investigar en detalle su alcance y naturaleza.

Martín-Loeches et al, (2011) aportan valores de permeabilidad en las calizas jurásicas entre 1.5 y $0.014 \mathrm{~m} / \mathrm{d}$ y en las cretácicas entre 0.5 y $0.045 \mathrm{~m} / \mathrm{d}$; aunque estos rangos de valores son normales en acuíferos carbonatados (Domenico \& Schwartz, 1998; Fetter 2001; Pulido, 1998 y 2001) resultarían superiores con mayor número de datos en diferentes puntos de las unidades estudiadas. El único valor que se posee en la zona para las Utrillas es de $0.068 \mathrm{~m} / \mathrm{d}$ y se encuentra en el rango indicado para arenas arcillosas por Fetter (2001).

El agua subterránea en todas las unidades descritas es $\mathrm{HCO}_{3}$-Ca-Mg con conductividades muy regulares próximas a los $600 \mu \mathrm{s} / \mathrm{cm}$ salvo en los puntos influenciados por las arcillas del Keuper donde se registran hasta $780 \mu \mathrm{s} / \mathrm{cm}$ (Martín-Loeches, 2008b). La presencia de $\mathrm{NO}_{3}^{-}$es constante, oscilando en los puntos de descarga regional entre 10 y $19 \mathrm{mg} / 1$. No se ha registrado de forma continua ningún parámetro hidroquímico en los manantiales de descarga de los macizos cretácicos, aunque hemos de esperar variaciones notables con la llegada del agua procedente de los conductos kársticos mayores tras las precipitaciones (Pulido, 2001).

La precipitación alcanza en esta región entre 500 y $600 \mathrm{~mm}$ en años medios constituyendo los ríos Dulce y Henares el drenaje principal de los acuíferos carbonatados que estudiamos.

A partir de la descomposición de hidrogramas de la estación de aforos 3254 (Fig. 2), y considerando $87.5 \mathrm{~km}^{2}$ de superficie vertiente a la citada estación de aforos, se estima que la infiltración eficaz oscila entre un 14 y un $20 \%$ de las precipitaciones de años medios a húmedos respectivamente.

Durante los estiajes rigurosos algunos tramos del río Dulce sobre los materiales de edad Jurásico se encuentran secos (Fig. 2) con la única aportación continua del manantial situado a $983 \mathrm{msnm}$ en el propio cauce (Fig. 4). El agua de los manantiales de Estriégana y Sauca, parece sufrir infiltración en la unidad Pelegrina-Torremocha Norte cerca de la localidad de Jodra del Pinar (Fig. 2) aunque no se ha

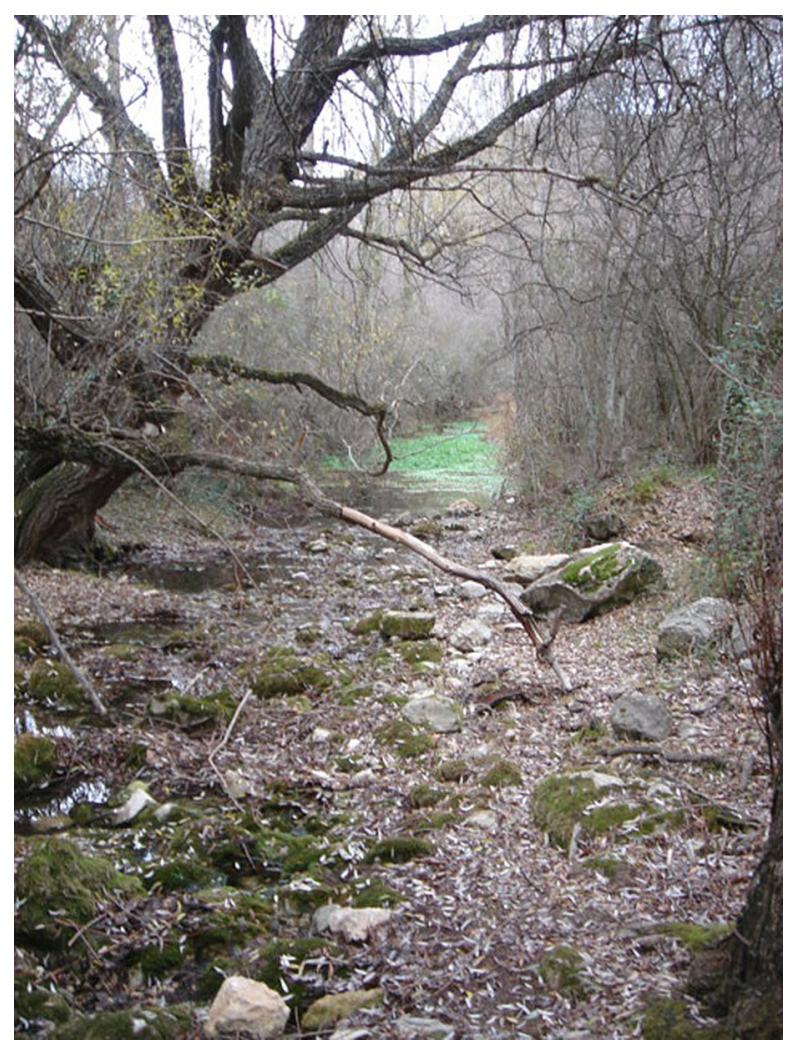

Fig. 4.-Manantial en el cauce seco del río Dulce a $983 \mathrm{msnm}$ (fotografía realizada en octubre de 2006).

localizado un sumidero claro; más bien parece producirse una entrada difusa del agua a lo largo de un tramo corto del cauce. A partir de los macizos cretácicos, en el entorno de la localidad de La Cabrera, se mantiene en el río Dulce un caudal mínimo.

\section{Análisis e interpretación de los resultados}

En la Tabla 2 se muestran los resultados de edades aparentes de las aguas subterráneas obtenidos bajo diferentes modelos, tras la aplicación del programa del USGS (USGS, 2013). La Fig. 5 es una representación gráfica de los mismos resultados.

En primer lugar se debe valorar si las concentraciones de los gases medidos corresponden a los aportes atmosféricos originales o por el contrario han sufrido alteraciones que deban tenerse en cuenta en las interpretaciones. En este sentido señalamos los siguientes puntos:

1) Los CFCs pueden degradarse en ambiente anaerobio (IAEA, 2006), sin embargo las medidas en 


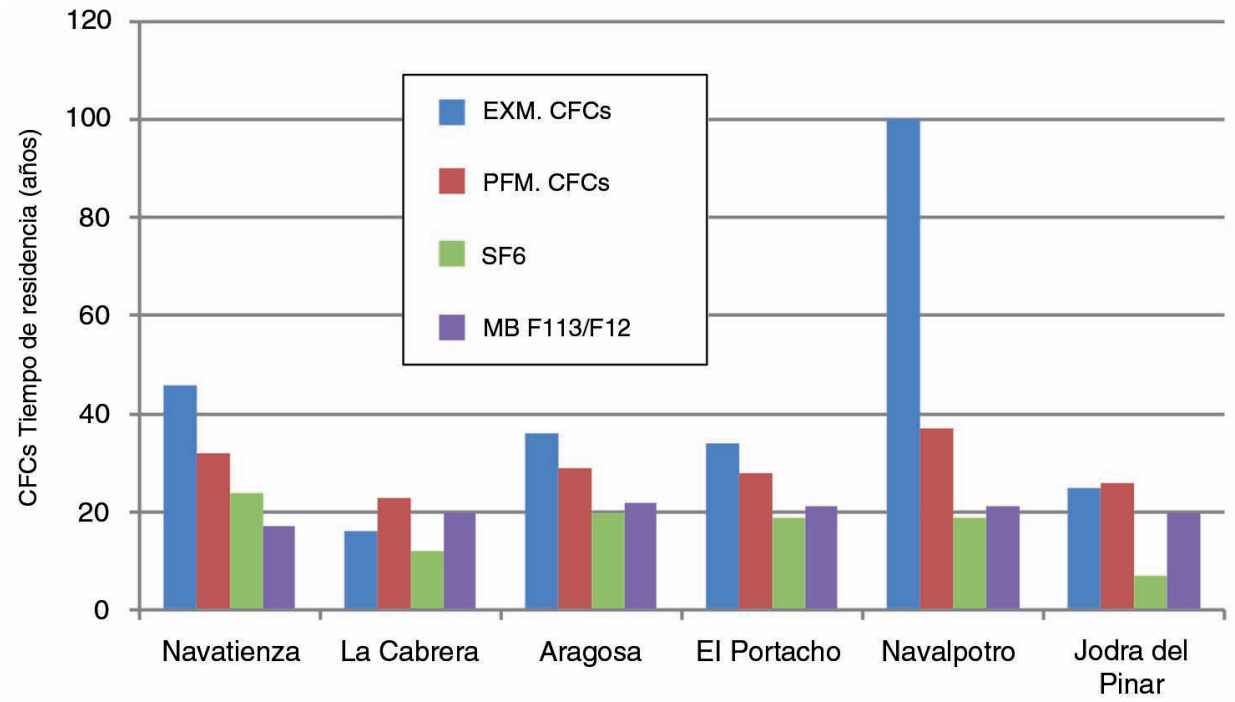

Fig. 5.-Edades aparentes obtenidas (tiempos de residencia) según diferentes modelos y trazadores. EXM=Modelo exponencial; PFM=Modelo flujo pistón; MB=Mezcla binaria (sólo fracción “joven”).

campo de oxígeno disuelto en el agua de los puntos muestreados superaba en todos los casos los 4 ppm indicando condiciones aerobias (Yager et al., 2013).

2) Salvo para la muestra F, se estima un espesor de zona no saturada en la recarga superior a los 30 metros (Fig. 3), valor a partir del cual Cook et al. (2006) indican que las edades estimadas pueden presentar un exceso de entre 8 y 12 años; al tratarse de acuíferos con un notable grado de fracturación, se ha considerado que el mecanismo de incorporación del agua de infiltración a la zona saturada es rápido y no hemos aplicado ninguna corrección (Gooddy et al., 2006; Darling et al., 2012).

3) La posibilidad de exceso de aire en las muestras no parece muy probable teniendo en cuenta los relativamente elevados valores de edad aparente obtenidos a partir de los CFCs y al hecho de que las edades derivadas del contenido en $\mathrm{SF}_{6}$ y las interpretadas por los CFCs son muy diferentes.

4) Las dataciones con $\mathrm{SF}_{6}$ son en todos los casos, menores que las obtenidas con los CFCs (Tabla 2); sus valores extremos $(0,7$ y $2,4 \mathrm{fmol} / \mathrm{L})$ lo son también en los CFCs, a excepción de La Cabrera, pero no responden a un factor de proporción único.
Si existiese exceso de aire afectaría igualmente a los contenidos de CFCs, con lo que las diferencias se deben a otros factores. Estas edades podrían explicarse por la incorporación de $\mathrm{SF}_{6}$ al agua subterránea procedente del sustrato carbonatado, que se suma al $\mathrm{SF}_{6}$ atmosférico, algo que se ha señalado por distintos autores en acuíferos de naturaleza semejante (Busenberg \& Plummer, 2006; Darling et al., 2012). Estas apreciaciones indican que los datos de $\mathrm{SF}_{6}$ no deben tenerse en cuenta en las interpretaciones.

5) La Cabrera (muestra B) presenta contaminación en CFC-11 (Tabla 2), lo que significa que existe una entrada de este compuesto diferente a la atmosférica (hay más CFC-11 que el que le correspondería en equilibrio con la atmósfera). No es contaminación por exposición de la muestra al aire actual, que afectaría a los otros compuestos. Según Cook et al. (2006), algunas fuentes de CFC son las aguas residuales procedentes de fosas sépticas, los basureros o las pérdidas del alcantarillado. Según este dato puede que a La Cabera lleguen efluentes procedentes de las aguas residuales de Algora donde se vierten al subsuelo con un mínimo tratamiento. Se recogieron dos muestras por cada punto de agua, obteniéndose los mismos resultados; no obstante se deben realizar otras determinaciones 
para confirmar esta contaminación. Si el agua está contaminada en un CFC pero no en los otros, como es el caso, la datación es posible (Plummer et al., 1993).

En relación a la interpretación de los datos, en términos generales las muestras con menor concen-

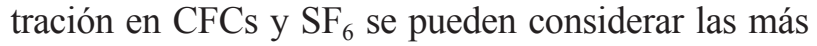
antiguas (muestras A y E, ver Tabla 1), teniendo en cuenta las curvas históricas de concentración de estos elementos en la atmósfera (Fig. 1), y aunque con ellas se puede llegar a algunas interpretaciones en relación al flujo en los acuíferos, es preferible realizar una interpretación bajo un modelo de mezcla que se ajuste a las condiciones del muestreo y a las características hidrodinámicas del acuífero (Darling et al, 2012).

Una forma de conocer el ajuste de las muestras a los modelos de mezcla posibles es representando los datos en gráficas binarias de concentración de un $\mathrm{CFC}$ respecto de otro o respecto del $\mathrm{SF}_{6}$ (Darling et al., 2012). Teniendo en cuenta la tendencia decreciente de la concentración en la atmósfera de los CFCs, las combinaciones CFC-113 versus CFC-12 y especialmente la CFC-11 versus CFC-12, presentan ambigüedades. El único gráfico que no ofrece problemas es el CFC-12 versus $\mathrm{SF}_{6}$, ya que la curva que representa el flujo pistón no se cruza con la línea que marca la mezcla binaria. Gooddy et al. (2007) diferencian la existencia de hasta 3 modelos de mezcla a partir de la representación en un gráfico de este tipo de muestras procedentes de una única trayectoria de flujo en un acuífero del Sur de Inglaterra. En nuestro caso, al distorsionarse las concentraciones de $\mathrm{SF}_{6}$ por aportes del sustrato, se invalida su uso en las interpretaciones. Utilizamos en su lugar la gráfica CFC-113 versus CFC-12, que mostramos en la Fig. 6, que aunque presenta cierta ambigüedad a partir de los últimos años de la década de los 80 , puede ofrecernos resultados más completos.

Vemos en la Fig. 6, que todas las muestras se encuentran siempre sobre alguna línea de mezcla, por encima de la línea que marca el flujo pistón.

Descartado el flujo pistón, los resultados pueden interpretarse como mezclas exponenciales o como mezclas binarias de edad. La mezcla exponencial aporta un único valor de tiempo de residencia (Tabla 2) en el que tiene más peso la señal del agua que se encuentra en mayor proporción, mientras que la interpretación según el modelo binario aporta información en relación a una mezcla simple de agua de dos diferentes edades. Así, a la muestra E, Navalpotro, se le asigna un valor de 100 años según el modelo exponencial o se interpreta, según el modelo de mezcla binaria, como una mezcla entre un 30\% de agua post- 1950 y un $70 \%$ de agua pre 1950 . Con el modelo de mezcla binaria se cuantifica la proporción de esa mezcla y se aporta un valor de edad de la fracción "joven". Ni en la mezcla exponencial ni en la binaria las edades aportadas son absolutas (Tabla 2).

Por la naturaleza carbonatada de los acuíferos estudiados, estos deben previsiblemente albergar al menos dos porosidades que derivan en flujos del agua subterránea que transcurren por vías diferentes; una lenta, a través de fracturas de pequeño tamaño y de la matriz de los bloques en los que se encuentra dividido el sustrato (especialmente en las unidades cretácicas) y otra rápida por los conductos kársticos mayores. Consideramos más ajustada por tanto la interpretación de los resultados a través de una mezcla binaria entendiendo que coexisten en las muestras al menos dos fracciones de edad, una de las cuales correspondería a aguas libres de CFCs que llegan al punto de muestreo por la vía lenta y la otra a agua "joven", que viaja por los conductos kársticos que aunque proceda de múltiples entradas, se homogeniza en agua de infiltración relativamente reciente.

Teniendo en cuenta estas apreciaciones, llegamos a las siguientes interpretaciones sobre las muestras analizadas, que exponemos de forma separada por unidades acuíferas:

1) Navalpotro (muestra E) y Navatienza (muestra A) son dos pozos perforados en la unidad Torremocha-Pelegrina Sur, de edad Jurásico (Fig. 2 y corte III-III' de la Fig. 3). Un 70\% del agua en Navalpotro es anterior a 1950, mientras que Navatienza, presenta mayor proporción de agua "joven" o post 1950 (Fig. 6). Esta diferencia puede deberse a una mayor llegada de agua subterránea de infiltración relativamente reciente a Navatienza, procedente de áreas al Sur de Torremocha del Campo (siempre en el material Jurásico), que a Navalpotro donde según el modelo propuesto existe menor gradiente y menor superficie de afloramiento del material 


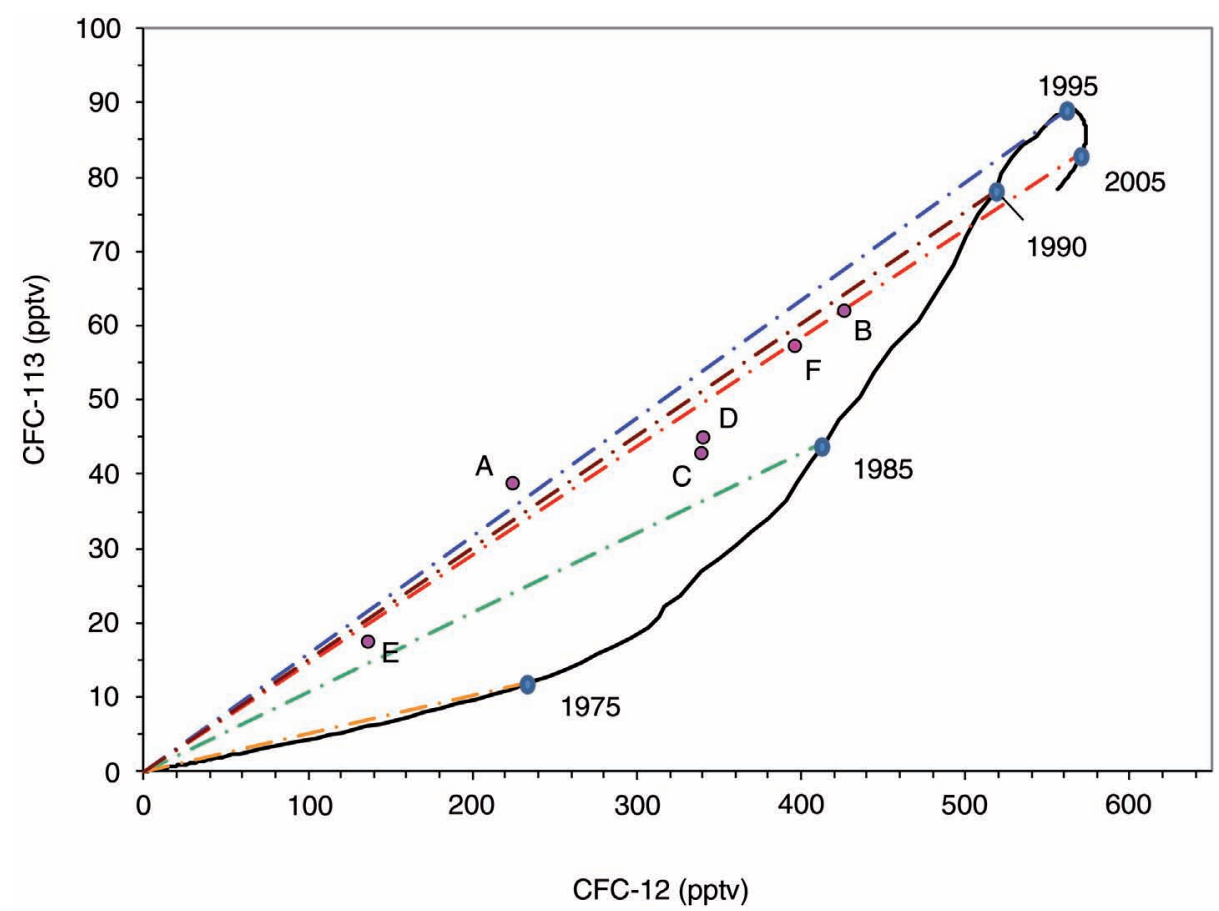

Fig. 6.-Representación de las muestras (puntos rosas) sobre una gráfica de concentraciones de CFC-113 respecto a CFC-12. Línea negra continua: flujo pistón; líneas discontinuas: mezclas binarias según diferentes años

Jurásico en condiciones saturadas, en la dirección supuesta del flujo (entre Navalpotro y Tortonda; ver Fig. 2). La elevada proporción en ambas muestras de agua anterior a 1950 es congruente con un flujo lento en estas zonas y por lo tanto con los gradientes estimados y con los bajos valores de la permeabilidad deducidos de los ensayos de bombeo.

2) Jodra del Pinar (muestra F) es un pozo que se encuentra en el extremo Este de la unidad calcárea jurásica de Torremocha-Pelegrina Norte (Fig. 2). Registra un porcentaje de agua "joven" cercano al $80 \%$ (Fig. 6) Este dato podría apoyar la idea de que el río Dulce, próximo a Jodra, estuviera perdiendo agua a favor del acuífero en esa zona, como propone el modelo de flujo de la Fig. 2. El gradiente aquí, además, es algo más elevado que en otras zonas (como por ejemplo en la zona de Navalpotro de la Unidad Pelegrina-Torremocha Sur), lo que favorece la renovación del agua.

3) El manantial de La Cabrera (muestra B) de la Unidad cretácica Algora-La Cabrera, presenta un
$86,2 \%$ de agua con CFCs, lo que nos indica que una parte muy importante de su caudal de base (que representa al mneos un 50\% de su descarga) está formado por agua joven o post-1950. El comportamiento hidrodinámico de este manantial es compatible con la existencia de una porosidad múltiple, al menos triple. La interpretación de los contenidos en CFC según la mezcla binaria no puede reflejar esta complejidad y menos aun obviamente el modelo exponencial a partir del cual se llega a un valor único de 16 años.

4) Aragosa (muestra C) y El Portacho (muestra D) son dos manantiales de descarga regional de la unidad cretácica de Aragosa (Fig. 2) y sus resultados son muy semejantes, con edades del entorno de 20 años para la fracción joven de ambas muestras, que se cifra en un $75 \%$ de su volumen (Fig. 6). Esta proporción indica, como en el caso de La Cabera, un importante aporte de aguas jóvenes infiltradas en las zonas de notable gradiente hidráulico que las precede (ver Fig. 2). A La Cabrera deben llegar aguas más veloces que a estos dos manantiales en virtud de su mayor 
proporción de aguas jóvenes y porque el valor medio de edad del Portacho y de Aragosa, según el modelo exponencial, es notablemente superior (Tabla 2 y Fig. 5). El Portacho y Aragosa son manantiales de descarga de un sistema calcáreo de menor superficie aflorante que el de Algora-La Cabrera y según el modelo de flujo propuesto con mayores gradientes hidráulicos, lo que está en consonancia con un registro de aguas jóvenes muy importante.

\section{Discusión y conclusiones}

Al no disponerse de datos de los trazadores medidos en las zonas de descarga de las unidades jurásicas, ni en las zonas de recarga en las cretácicas no es posible llegar a valoraciones completas en relación al flujo del agua subterránea. Los datos sí apoyan el conocimiento previo deducido por otras vías.

A excepción del agua recogida en Jodra del Pinar, las muestras de las unidades cretácicas poseen mayor proporción de aguas jóvenes que las recogidas en las jurásicas, lo que puede indicarnos que los macizos cretácicos poseen mayor permeabilidad y presencia de vías de circulación del agua subterránea más rápidas, lo que está de acuerdo con el modelo conceptual propuesto. Esta suposición no se encuentra refrendada por los datos de conductividad hidráulica medidos en los sondeos, pero hay que tener en cuenta que estos no son representativos del comportamiento general de unos acuíferos de gran heterogeneidad y anisotropía. En este sentido los trazadores constituyen una herramienta discriminante.

Aun considerando que los datos son escasos y se debe seguir investigando, ha quedado claro que las interpretaciones de los trazadores no derivan en valores de edad que se ajusten a la complejidad natural de estos sistemas. Esta conclusión es clara en el caso de La Cabrera ya que es en este punto donde mayor información hidrodinámica se posee y ha podido ser comparada, pero se daría con toda probabilidad en el resto de los puntos de agua.

La elevada edad de las fracciones jóvenes de todos los puntos (siempre por encima de los 17 años) indica que se recogen sin un volumen significativo del agua que descarga de forma rápida tras las precipitaciones importantes y que circula por los conductos kársticos mayores. Esta fracción representa una parte pequeña del almacenamiento en este tipo de acuíferos (menos del 4\%, según Worthington, 2000) y se debe estudiar a través del uso de técnicas de trazado con químicos solubles colorantes o con trazadores sólidos en suspensión. A su vez este dato muestra que debe ser importante el flujo que circula a través de la matriz y de las fracturas más pequeñas y medias.

En términos generales los CFCs han sido eficientes para confirmar y cuantificar mezclas de aguas en sistemas binarios, lo que ha supuesto la base de su utilidad en estos acuíferos, suficiente para apoyar un determinado sistema de flujo. Los resultados obtenidos, son coherentes con un modelo de escaso gradiente en la recarga y con la existencia de al menos una doble porosidad en todos los acuíferos. Los CFCs han servido además para poner de manifiesto la posible presencia de contaminación urbana en las muestras.

Además de la ausencia de puntos de agua susceptibles de muestrearse en algunas áreas de estos acuíferos, existen otros elementos que deben considerarse para realizar una mejor interpretación de los contenidos en CFCs y $\mathrm{SF}_{6}$; caben destacarse un mejor conocimiento de la forma del flujo en la zona no saturada y la verificación de la ausencia de ambiente reductor en algunas zonas profundas de estos macizos aunque activas en el flujo.

Por otro lado, se ha puesto de manifiesto, una vez más, lo importante de acompañar los valores de CFCs y $\mathrm{SF}_{6}$ con los de otros trazadores (Han, et al., 2006). El ${ }^{3} \mathrm{H}$ y la relación ${ }^{3} \mathrm{H} /{ }^{3} \mathrm{He}$, pueden ayudar a validar las edades aparentes obtenidas con los CFCs; el ${ }^{14} \mathrm{C}$ puede ser útil para estimar las fracciones de mezcla y la edad de la porción pre-1950 en las muestras, donde se ha establecido la existencia de mezclas; para calcular la temperatura en la recarga es importante además contar con medidas de gases como el $\mathrm{N}_{2}$ y el Ar (Lindsey, et al., 2003; Yager, et al., 2013).

Existen dos importantes puntualizaciones sobre el futuro del uso de los CFCs y el $\mathrm{SF}_{6}$ en estos acuíferos carbonatados, ambas relacionadas; en primer lugar, si se confirma que el sustrato carbonatado de estos macizos aporta $\mathrm{SF}_{6}$ al agua subterránea, lo que puede realizarse con mayores determinaciones, se invalidaría su uso como trazador. En segundo lugar y como consecuencia, cada vez será más difícil distinguir el modelo de mezcla al que puede ajustarse una muestra a partir de su contenido en CFC, elemento 
muy importante para su interpretación, ya que las curvas de concentración de CFCs en la atmósfera se encuentran decreciendo.

\section{AGRADECIMIENTOS}

El muestreo de CFCs y $\mathrm{SF}_{6}$ se realizó con la ayuda del proyecto de Investigación CGL2004-02179/BTE de la DGYCT y los trabajos posteriores recibieron el apoyo de la Junta de Comunidades de Castilla-La Mancha a través de la subvención GUGE002609. Los autores quisieran expresar su agradecimiento a Juan Antonio Calvo, Javier Temiño Vela y José Francisco García-Hidalgo Pallarés, del Departamento de Geología, Geografía y Medio Ambiente de la Universidad de Alcalá por su apoyo durante los trabajos realizados y a E. Busenberg, del Servicio Geológico de los Estados Unidos y a H. Oster del laboratorio de CFC de Wachenheim (Alemania), por sus comentarios y aportaciones en la interpretación de los datos. Agradecemos a los revisores de este trabajo sus importantes aportaciones.

\section{Referencias}

Adell, F.; Tena-Dávila, M. \& Gonzalez, F. (1981a). Mapa Geológico de España. Hoja n 461 "Siguenza”. IGME, Madrid.

Adell, F.; Bascones, L.; Martín, D. \& Martínez-Álvarez, F. (1981b). Mapa Geológico de España. Hoja ${ }^{\circ} 488$ "Ablanque". IGME, Madrid.

Ministerio de Industria, Turismo y Comercio de España (2014). Archivo Técnico de Hidrocarburos. https:// geoportal.minetur.gob.es/ATHv2/welcome.do.

Atkinson, T.C. \& Smart, P.L. (1981). Artificial tracers in hydrogeology. In: A survey of British hydrogeology, London, Royal Society, 173-190.

Bascones, L. \& Martínez, F. (1981). Mapa Geológico de España. Hoja n 462 “Maranchón”. IGME, Madrid.

Busenberg, E. \& Plummer, L.N. (1992). The use of chlorofluorocarbons $(\mathrm{CCl} 3 \mathrm{~F}$ and $\mathrm{CCl} 2 \mathrm{~F} 2)$ as hydrologic tracers and age-dating tools: the alluvium and terrace system of Central Oklahoma. Water Resources Research, 28: 2257-2283. http://dx.doi.org/10.1029/ 92WR01263.

Busenberg, E. \& Plummer, L.N. (2000). Dating young groundwater with sulfur hexafluoride: natural and anthropogenic sources of sulfur hexafluoride. Water Resources Research, 36: 3011-3030. http://dx.doi. org/10.1029/2000WR900151.

Busenberg, E. \& Plummer, L.N. (2006). Potential use of other atmospheric gases. In: Use of Chlorofluorocarbons in Hydrology: A Guidebook. IAEA, Viena, 183-189.

Busenberg, E. \& Plummer, L.N. (2008). Dating groundwater with trifluoromethyl sulfurpentafluoride (SF5CF3), sulfur hexafluoride (SF6), CF3Cl (CFC-13), and CF2C12 (CFC-12). Water Resources Research, 44: W02431. http://dx.doi.org/10.1029/2007WR006150.
Busenberg, E.; Plummer, L.N.; Cook, P.G.; Solomon, D.K.; Han, L.F.; Gröning, H. \& Oster, H. (2006). Sampling and analytical methods. In: Use of Chlorofluorocarbons in Hydrology: A Guidebook. IAEA, Viena, 199-217.

CHT (2013). Página web de la Confederación Hidrográfica del Tajo. http://www.chtajo.es/Paginas/default. aspx.

Cook, P.G.; Plummer, L.N.; Solomon, D.K.; Busenberg, E. \& Han, L.F. (2006). Effects and processes that can modify apparent CFC age. In: Use of Chlorofluorocarbons in Hydrology: A Guidebook. IAEA, Viena, 31-56.

Cook, D.K. \& Solomon, D.K. (1995). Transport of trace gases to the water table: Implications for groundwater dating with chlorofluorocarbons and krypton- 85 . Water Resources Research, 31: 263-270. http:// dx.doi.org/10.1029/94WR02232.

Cook, P.G. \& Solomon, D.K. (1997). Recent advances in dating young groundwater: Chlorofluorocarbons, 3H/3He and 85Kr. Journal of Hydrology, 191: 245-265. http://dx.doi.org/10.1016/S0022-1694(96)03051-X.

Darling, W.G.; Gooddy, D.C.; MacDonald, A.M. \& Morris, B.L. (2012). The practicalities of using CFCs and SF6 for groundwater dating and tracing. Applied Geochemistry, 27 (9): 1688-1697. http://dx.doi.org/10.1016/j. apgeochem.2012.02.005.

Domenico, P.A. \& Schwartz, F.W. (1998). Physical and Chemical Hydrogeology. John Wiley \& Sons, New York, 824 pp.

Fetter, C.W. (2001). Applied Hydrogeology (4th ed.), Prentice-Hall, Upper Saddle River, New Jersey, 598 pp.

Ford, D. \& Williams, P. (2007). Karst Hydrogeology and Geomorphology. Wiley, 576 pp. http://dx.doi.org/ $10.1002 / 9781118684986$.

García-Hidalgo, J.F.; Martín-Loeches, M.; Gonzalez, J.A.; Aguilar, M. \& García-Quintana, A. (2008). Geología, hidrogeología y paisaje en el Parque Natural del Barranco del Río Dulce (Guadalajara, España). In: Geología de Guadalajara (Calonge, A. \& Rodriguez, M., Eds.). Obras colectivas, Ciencias 03, Universidad de Alcalá de Henares, 269-290.

Goldscheider, N. \& Drew, D. (2007). Methods in Karst Hydrogeology. Taylor \& Francis, London, 264 pp.

Gooddy, D.C.; Darling, W.G.; Abesser, C. \& Lapworth, D.J. (2006). Using chlorofluorocarbons (CFCs) and sulphur hexafluoride (SF6) to characterise groundwater movement and residence time in a lowland Chalk catchment. Journal of Hydrology, 330 (1-2): 44-52. http://dx.doi.org/10.1016/j.jhydrol.2006.04.011.

Goy, A.; Gómez, J. \& Yébenes, A. (1976). El Jurásico de la Rama Castellana de la Cordillera Ibérica (Mitad Norte). Unidades Litoestratigráficas. Estudios Geológicos, 32: 261-283.

Han, L.F.; Gröning, M.; Plummer, L.N. \& Solomon, D.K. (2006). Comparison of the CFC technique with other techniques $(3 \mathrm{H}, 3 \mathrm{H} / 3 \mathrm{He}, 85 \mathrm{Kr})$. In: Use of 
Chlorofluorocarbons in Hydrology: A Guidebook. IAEA, Viena, 191-197.

IAEA (2006). Use of Chlorofluorocarbons in Hydrology: A Guidebook. IAEA, STI/PUB/1238. 277 pp.

IGME (2013). Unidades hidrogeológicas de España. http:// www.igme.es/.

Lindsey, B.D.; Phillips, S.W.; Donnelly, C.A.; Speiran, G.K.; Plummer, L.N.; Bohlke, J.K.; Focazio, M.J.; Burton, W.C. \& Busenberg, E. (2003). Residence times and nitrate transport in ground water discharging to streams in the Chesapeake Bay Watershed. U.S. Geological Survey Water-Resources Investigations Report 03-4035, 201 pp.

Martín-Loeches, M. (2008a). Hidrogeología de los acuíferos carbonatados con influencia en el parque natural del Barranco del río Dulce, Guadalajara. Actas del IX Simposio De Hidrogeología. Asociación Española De Hidrogeólogos. Elche.

Martín-Loeches, M. (2008b). Hidrogeoquímica y contenido en isótopos ambientales de las aguas subterráneas de los acuíferos carbonatados con influencia en el parque natural del Barranco del río Dulce, Guadalajara. Actas del IX Simposio De Hidrogeología. Asociación Española De Hidrogeólogos. Elche.

Martín-Loeches, M.; Díaz Alcaide, S. \& Martinez, P. (2011). Las aguas subterráneas en el entorno del Parque Natural del Barranco del Río Dulce. Características generales, modelo de flujo conceptual y elaboración de un modelo numérico como herramienta de gestión. JCCM, Informe interno proyecto GUGE002609. xx pp.

Plummer, L.N.; Michel, R.L.; Thurman, E.M. \& Glynn, P.D. (1993). Environmental tracers for age-dating young ground water. In: Regional Ground-water Quality (Alley, W.M., Ed.), Van Nostrand Reinhold, 255-294.

Plummer, L.N. \& Busenberg, E. (1999). Chlorofluorocarbons. In: Environmental Tracers in Subsurface Hydrology, Kluwer, Dordrecht, 441-478.

Plummer, L.N.; Böhkle, J.K. \& Busenberg, E. (2003). Approaches for ground-water dating. In: Residence times and nitrate transport in groundwater discharging to streams in the Chesapeake Bay Watershed. U.S. Geological Survey Water-Resources Investigations Report 03-4035, 12-24.
Pulido, A. (1998). Principales rasgos hidrogeológicos de los macizos kársticos andaluces. In: Karst en Andalucía (Durán, J.J. \& López Martinez, J., Eds.), 49-53.

Pulido, A. (2001). Investigación y exploración de acuíferos kársticos. Boletín Geológico y Minero, 112: 65-76.

Quinlan, J.F.; Smart, P.L.; Schindel, G.M.; Alexander, E.C. Jr.; Edwards, A.J. \& Smith, A.R. (1992). Recommended administrative/regulatory definition of carbonate aquifers, principles for classification of carbonate aquifers, practical evaluation of vulnerability of karst aquifers, and determination of optimum sampling frequency at springs. In: Proceedings of the third conference on hydrogeology, ecology, monitoring, and management of groundwater in karst terrains, Dublin, Ohio, Water Well Journal Publishing Company, 573-635.

Ríos, L.M.; Barettino, D.; Racero, A. \& Galera, J.M. (2002). Mapa Geológico de España. Hoja n ${ }^{\circ} 487$ "Ledanca". IGME, Madrid.

Smart, P.L. \& Hobbs, S.L. (1986). Characterisation of carbonate aquifers: a conceptual base. In: 1st Environmental Problems in Karst Terranes and their Solutions Conference (Bowling Green, Kentucky), National Water Well Association, Dublin, Ohio, 1-14.

USGS (2013). USGS spreadsheet program for preliminary evaluation of CFC data. http://water.usgs.gov/.

White, W.B. (1969). Conceptual models for carbonate aquifers. Ground Water, 7: 15-21. http://dx.doi.org/ 10.1111/j.1745-6584.1969.tb01279.x.

Worthington, S.R.H.; Davies, G.J. \& Ford, D.C. (2000). Matrix, fracture and channel components of storage and flow in a Paleozoic limestone aquifer. In: Groundwater flow and contaminant transport in carbonate aquifers (Wicks, C.M. \& Sasowsky, I.D., Eds.), Rotterdam, Balkema, 113-128.

Yager, R.M.; Plummer, N.; Kauffman, L.J.; Doctor, D.H.; Nelms, D.K. \& Schlosser, P. (2013). Comparison of age distributions estimated from environmental tracers by using binary-dilution and numerical models of fractured and folded karst: Shenandoah Valley of Virginia and West Virginia, USA. Hydrogeology Journal, 21: 1193-1217. http://dx.doi.org/10.1007/ s10040-013-0997-9. 